respiratory failure. Bronchiolitis was the most frequent condition (28\%), followed by upper airway obstruction (15.2\%), acute cardiogenic pulmonary edema (15.2\%) and pneumonia (14.4\%). CPAP was the respiratory mode more used.NIV success rate was $67.5 \%$ : $2.3 \%$ in the first hour, $32 \%$ between $1^{\text {st }}-12^{\text {th }}$ hour and $23.3 \%$ between $12^{\text {th }}-24^{\text {th }}$ hour. Failure rate was greater among patients with type I respiratory failure (34.9\%) and acute respiratory distress syndrome $(66.7 \%)$. A lower heart and respiratory rate at 6 hours were associated with NIV failure $(p<0.05)$.

Conclusions NIV is a useful and increasingly used ventilatory mode in PICU. Type I respiratory failure, decrease in heart rate and respiratory rate at 6 hours were risk factors for NIV failure. More studies involving predictive factors in children are still needed.

\section{NEBULISED ILOPROST AND NONINVASIVE RESPIRATORY SUPPORT AS A FIRST TREATMENT FOR HYPOXAEMIC RESPIRATORY FAILURE IN EX-PRETERM INFANTS: PRELIMINARY EXPERIENCE}

doi:10.1136/archdischild-2012-302724.0992

'M Piastra, 'D de Luca, 'D Pietrini, 'E Luca, 'L Marzano, ${ }^{2} \mathrm{G}$ de Rosa, 'F Visconti, ${ }^{3} \mathrm{~F}$ Caliandro, ' $\mathrm{G}$ Conti. ' $E$ Emergency Department, Pediatric ICU; ' 2 Department of Pediatrics, Pediatric Cardiology, Catholic University of the Sacred Heart, A. Gemelli Hospital, Roma, Italy; ' ${ }^{3}$ Department of Cardio-Thoracic Anaesthesia, St. George's Hospital NHS, London, $U K$

Objective To describe a series of ex-preterm infants admitted to pediatric intensive care unit because of acute hypoxaemic respiratory failure complicated by pulmonary hypertension who were treated electively combining noninvasive ventilation (NIV) and nebulized iloprost (nebILO).

Methods Open uncontrolled observational study, Pediatric Intensive Care Unit, University Hospital.

Measurements and Main Results Ten formerly preterm infants with acute hypoxaemic respiratory failure and pulmonary hypertension, of whom 8 had moderate to severe bronchopulmonary dysplasia. Median age and body weight were 6.0 (2.75-9.50) months and $4.85(3.32-7.07) \mathrm{kg}$, respectively. We observed a significant early oxygenation improvement in terms of $\mathrm{PaO}_{2} / \mathrm{FiO}_{2}$ increase $(p=0.001)$ and respiratory rate reduction $(p=0.01)$. Hemodynamic also improved, as shown by heart rate $(\mathrm{p}=0.002)$ and pulmonary arterial pressure systolic/systolic systemic pressure (PAPs/SSP) ratio reduction ( $p=0.0137)$. NebILO was successfully weaned in positive response cases: 4 infants were discharged on oral sildenafil. Three patients failed noninvasive modality and needed invasive mechanical ventilation; hypoxic-hypercarbic patients were most likely to fail noninvasive approach. Only one patient requiring invasive ventilation died and surviving babies had a satisfactory 1-month postdischarge follow-up.

Conclusions The noninvasive approach combining NIV and nebILO for ex-preterm babies with respiratory failure and pulmonary hypertension resulted to be feasible and quickly achieved significant oxygenation and hemodynamic improvements.

\section{IMPLEMENTATION OF VENTILATION POLICY IN A PICU}

doi:10.1136/archdischild-2012-302724.0993

A Duyndam, B van Driel, RJ Houmes, D Tibboel, E Ista. Intensive Care Kinderen, Erasmus MC - Sophia Childrens Hospital, Rotterdam, The Netherlands

Background and Aims Pediatric intensive care units (PICU) worldwide use different ventilators with a wide variety of ventilation modes. As an unambiguous international ventilation guideline, we developed one. After implementation we evaluated to what extent physicians adhered to the new guideline.

Method We developed a ventilation guideline accounting for two groups: 1) heterogeneous lung disease, in which pressure control is the preferred mode; 2) homogeneous lung disease, in which pressureregulated volume control is preferred. The guideline was implemented in October 2008. We performed an uncontrolled, retrospective before-after design with a pre-test from January to July 2008 (T0) and two post-tests: May-November 2009 (T1); May-November 2010 (T2). All patients on conventional invasive mechanical ventilation during these periods were included. Outcome measure was the percentage of physicians' adherence to the ventilation protocol. We measured this by describing the ventilation mode on the first hour on the day of admission and the cause of respiratory failure, to distinguish in which group this patient belonged.

Results In group 1, the T0 adherence percentage was 79\% (67/85). Adherence percentages after implementation of the guideline were $71 \%(51 / 72)$ and $84 \%(46 / 55)$ for respectively T1 and T2. For group 1 , adherence in period T2 was slightly better $(\mathrm{p}=0.092)$ than that in period T1. In group 2, adherence percentages rose statistically significantly from $66 \%$ at T0 (62/93) to $78 \%(79 / 101)$ and $84 \%(85 / 101)$ $(\mathrm{p}=0.015)$

Conclusion Implementation of a new ventilation guideline increased guideline adherence over time. Selection of the appropriate ventilation mode seems now clearer for physicians.

\section{NON-INVASIVE VENTILATION (NIV) IN CHILDREN - ESTABLISHMENT OF A PEDIATRIC NIV SCORING SYSTEM}

doi:10.1136/archdischild-2012-302724.0994

K Bohn, S Gehring, R Huth, C Martin. Children's Hospital of the University Mainz, Mainz, Germany

Objectives Non-invasive ventilation (NIV) is being increasingly used in children with respiratory failure in order to avoid intubation and associated problems. We analyzed the efficiency of NIV in children and the outcome of our patients.

Methods In a retrospective study children who received NIV over the last 7 years were analyzed. Included were all children that had at least more than one hour of NIV and a cardiological disease or an infection of the airway. Patients were divided in subgroups according to their underlying disease. The following parameters were analysed: age, gender, weight, mode of NIV, hemodynamic and ventilatory status, blood gas analysis, days of hospitalisation and mortality rate.

Results 70 patients between the age of 1 day to 28 years that received NIV were analyzed. The study population consisted of: 35 cardiological patients (50\%) and 35 patient with an infection of the airway (50\%). Children that had to be intubated because of a respiratory failure were classified as nonresponders. The overall rate of responders was at least $79 \%$. Response correlated significantly with the Positive End-Expiratory Pressure (PEEP) values, $\mathrm{pCO}_{2}$ and $\mathrm{FiO}_{2}$ at 6 hours after initiation of NIV.

Conclusion NIV offers an effective and successful alternative to conventional mechanical ventilation of children with respiratory failure. Due to advances in the currently available equipment and NIV algorithms we could significantly improve the rate of responders. Based on our findings we established a pediatric NIV score helping to predict NIV success.

\section{IMPACT OF VENTILATOR-ASSOCIATED PNEUMONIA ON TREATMENT AND LENGTH OF STAY IN CRITICALLY PEDIATRIC PATIENTS WITH LOWER RESPIRATORY SYSTEM INFECTION}

doi:10.1136/archdischild-2012-302724.0995

S Stabouli, A Violaki, E Volakli, L Vogiatzi, K Skoumis, M Sdougka. PICU, Hippokration Hospital, Thessaloniki, Greece

Background and Aims Ventilator-associated pneumonia (VAP) may complicate the hospital course in critically ill children with 